\title{
Diversity of nontuberculous mycobacteria in eastern and southern China: a cross-sectional study
}

\author{
To the Editor:
}

Nontuberculous mycobacteria (NTM) are a heterogeneous group of widely distributed environmental bacteria [1]. Despite generally low pathogenicity in humans, NTM can cause various illnesses especially in HIV-infected patients or those with immunodeficiencies [2]. In recent years, NTM-related disease incidence has increased significantly [3] and, despite China's tuberculosis (TB) programme having more than halved TB prevalence over the past 20 years [4], population-based data indicate that the proportion of NTM among all mycobacterial patient isolates has increased from $11.1 \%$ to $22.9 \%$ [5], triggering public health concerns.

Notably, NTM prevalence varies greatly across China [6-8], as southern and eastern regions exhibit higher NTM prevalence than elsewhere. Although the exact reason for this phenomenon is unclear, researchers believe that higher humidity in China's southern and eastern regions creates a more suitable environment for promoting NTM habitation. Recently, worldwide epidemiological evidence has demonstrated that NTM species distribution differs significantly by region [1]. As NTM species differ markedly in pathogenicity and drug susceptibility profiles, understanding this diversity is thus essential in informing clinical treatment [9]. Unfortunately, the geographic diversity of NTM in China, especially in areas of high NTM prevalence, has never been systemically investigated. Therefore, we present here data to further characterise and compare the distribution of NTM subspecies in patient isolates from southern and eastern China.

Between January 2012 and December 2014, 938 and 512 NTM isolates were collected consecutively from two representative regional TB clinical centres: Guangzhou Chest Hospital in southern China and Shanghai Pulmonary Hospital in eastern China, respectively. Of 1450 strains, 858 (59.2\%) were isolated from inpatients and $592(40.8 \%)$ from outpatients. Moreover, 1293 strains (89.2\%) were cultured from sputum, 131 (9.0\%) from bronchoalveolar lavage fluid and 26 (1.8\%) from other clinical sources. All strains were classified into subspecies using multilocus sequence analysis [5]. Chi-squared tests were performed using SPSS 14.0 (SPSS Inc, Chicago, IL, USA) to compare the distribution of different NTM species between Guangzhou and Shanghai. Differences were considered to be statistically significant for $\mathrm{p}<0.05$.

Overall, the five most frequently isolated NTM belonged to the Mycobacterium abscessus complex (subspecies: M. abscessus, Mycobacterium massiliense and Mycobacterium bolletii; 605 isolates, 41.7\%), to the Mycobacterium avium complex (subspecies: M. avium and Mycobacterium intracellulare; 330 isolates, 22.8\%), and to the Mycobacterium gordonae (244 isolates, 16.8\%), Mycobacterium kansasii (179 isolates, $12.3 \%$ ) and Mycobacterium fortuitum (50 isolates, 3.4\%) species, respectively. These five groups accounted for $97 \%$ of all mycobacteria identified. In Shanghai, M. intracellulare was the most commonly isolated NTM, accounting for $31.3 \%$ of all NTM isolates compared to $9.7 \%$ in Guangzhou $(p<0.01)$. Moreover, the most frequently isolated species in Guangzhou, M. abscessus, was more prevalent there (25.9\%) than in Shanghai $(19.5 \%)(\mathrm{p}=0.01)$. M. massiliense was the second most frequently isolated NTM in Guangzhou and was isolated significantly more frequently there $(22.9 \%)$ than in Shanghai $(8.8 \%)(\mathrm{p}<0.01)$. In addition, M. gordonae was isolated in Guangzhou significantly more frequently $(22.5 \%)$ than in Shanghai $(6.4 \%)(\mathrm{p}<0.01)$, whilst a higher percentage of $M$. kansasii was isolated in Shanghai (18.6\%) than in Guangzhou (9.0\%) $(\mathrm{p}<0.01)$ (table 1).

@ERSpublications

Nontuberculous mycobacterial infections in China: regional predominance of $M$. intracellulare and M. abscessus http://ow.ly/vg3H306YH8c

Cite this article as: Pang Y, Tan Y, Chen J, et al. Diversity of nontuberculous mycobacteria in eastern and southern China: a cross-sectional study. Eur Respir J 2017; 49: 1601429 [https://doi.org/10.1183/ 13993003.01429-2016]. 


\begin{tabular}{|c|c|c|c|c|}
\hline \multirow[t]{2}{*}{ Species } & \multicolumn{3}{|c|}{ Isolates $^{\#}$} & \multirow[t]{2}{*}{ p-value ${ }^{\pi}$} \\
\hline & Shanghai & Guangzhou & Total & \\
\hline M. intracellulare & 160 (31.3) & 91 (9.7) & 251 (17.3) & $<0.01$ \\
\hline M. avium & $52(10.2)$ & $27(2.9)$ & 79 (5.4) & $<0.01$ \\
\hline M. abscessus & $100(19.5)$ & 243 (25.9) & $343(23.7)$ & 0.01 \\
\hline M. massiliense & 45 (8.8) & 215 (22.9) & $260(17.9)$ & $<0.01$ \\
\hline M. kansasii & $95(18.6)$ & $84(9.0)$ & 179 (12.3) & $<0.01$ \\
\hline M. gordonae & $33(6.4)$ & $211(22.5)$ & $244(16.8)$ & $<0.01$ \\
\hline M. fortuitum & $17(3.3)$ & 33 (3.5) & 50 (3.4) & 0.84 \\
\hline M. lentiflavum & $8(1.6)$ & $4(0.4)$ & $12(0.8)$ & 0.03 \\
\hline M. yongonense & $0(0.0)$ & $9(1.0)$ & $9(0.6)$ & 0.03 \\
\hline M. parascrofulaceum & $0(0.0)$ & $5(0.5)$ & $5(0.3)$ & 0.17 \\
\hline M. simiae & $0(0.0)$ & $4(0.4)$ & $4(0.3)$ & 0.30 \\
\hline Other & $2(0.4)$ & 12 (1.3) & $14(1.0)$ & 0.16 \\
\hline Total & 512 (100.0) & 938 (100.0) & 1450 (100.0) & - \\
\hline
\end{tabular}

Data are presented as $\mathrm{n}$ or $\mathrm{n}(\%)$ unless otherwise stated. " : Percentage values were calculated based on the sum of isolates from all species for each city. ": The p-value was calculated to compare the proportions of nontuberculous mycobacterial (NTM) species between Shanghai and Guangzhou.

We also analysed the distribution of various subspecies within the M. avium and M. abscessus complexes. Interestingly, there was no statistical difference in the percentage of $M$. intracellulare in $M$. avium complex organisms between Shanghai $(75.5 \%, 160 / 212)$ and Guangzhou (77.1\%, 91/118) ( $\mathrm{p}=0.11)$, whilst M. massiliense accounted for a higher percentage of $M$. abscessus complex organisms isolated in Guangzhou $(46.9 \%, 215 / 458)$ than in Shanghai $(31.0 \%, 45 / 145)(\mathrm{p}<0.01)$. In addition, we were the first to report on several rare, geographically restricted NTM species in China. Mycobacterium lentiflavum was observed in both Guangzhou (4 isolates, 0.4\%) and Shanghai (8 isolates, 1.6\%). In contrast, Mycobacterium yongonense (9 isolates, 1.0\%), Mycobacterium parascrofulaceum (5 isolates, $0.5 \%$ ) and Mycobacterium simiae (4 isolates, $0.4 \%$ ) were identified only in Guangzhou.

This study was the first to demonstrate that NTM distribution differs significantly across China. In agreement with a recent study by SHAO et al. [7], the predominant NTM of eastern China was $M$. intracellulare. Moreover, WANG et al. [6] demonstrated that 40\% of NTM in Beijing were M. intracellulare. After pooling our findings with the Beijing findings, $M$. intracellulare prevalence was observed to gradually but obviously increase with increasing latitude. Unlike in Shanghai, the most frequently observed NTM in Guangzhou was the M. abscessus complex, with a higher incidence than that detected in Taiwan (30.2\%), South Korea (26.7\%) and Beijing (29.5\%) [6, 10]. We postulate that diverse geographic NTM patterns in China may result from climate variability, where NTM have evolved to survive in species-specific environmental niches [11]. In addition, a US study has revealed that $M$. abscessus complex predominates in tap water derived from surface water [12]. Furthermore, biofilm formation is a successful survival strategy for NTM and rapidly growing mycobacteria (RGM), such as M. abscessus and M. Fortuitum, exhibit excellent biofilm-forming capabilities in aqueous environments, even in constantly running water [12]. As such, M. abscessus complex members are more likely to inhabit humid areas than other species. Furthermore, environmental temperature also influences the distribution of NTM type [13] and evidence collected over the past 20 years indicates that RGM are associated with high-temperature habitats [13]. We therefore hypothesise that M. abscessus complex organisms preferentially inhabit niches with higher humidity and temperature, thereby resulting in their high prevalence in southern China. Further analysis of environmental NTM diversity will provide additional evidence to explain the M. abscessus complex epidemic in southern China.

Another interesting finding of this study is the significantly higher prevalence of M. gordonae in Guangzhou compared to Shanghai. M. gordonae is generally regarded as a rarely-isolated, weak pathogen that occasionally causes serious disease in both immunocompetent and immunocompromised individuals. While it is difficult to rule out detection resulting from culture contamination, transient harmless patient colonisation, or patient infection by disease-causing organisms, the higher rate of isolation of $M$. gordonae in Guangzhou may ultimately be due to the humid local environment, which is a suitable habitat for this tap-water bacillus.

Notably, M. simiae complex has been shown to cause opportunistic infections in humans in different regions worldwide, with the exception of Asia $[1,14]$. Here, for the first time, we identified in China three M. simiae 
complex subspecies: M. lentiflavum, M. parascrofulaceum and M. Simiae. While this could be due to the limited data available on this topic, the generally low prevalence of $M$. simiae complex in China may also be attributed to their inability to persist in human airways or a scarce environmental presence [1].

As conventional biochemical identification is routinely performed only in prefectural laboratories, further subspecies-level identification using molecular methods is difficult for other laboratories. Considering the significant differences in treatment regimen for various NTM infections, the current study may provide important hints to help clinicians select effective treatments in resource-limited settings in China.

An important shortcoming of our study is that patient data are not available, due to a high proportion of outpatients. In addition, the scarcity of laboratories is another shortcoming and further research will be needed using data from various laboratories to gain a detailed picture regarding geographic diversity of NTM across China.

In conclusion, this study illustrates that the NTM species distribution differs by region in China, with M. intracellulare predominating in eastern China while $M$. abscessus predominates in southern China. The geographic diversity of NTM in China may reflect the NTM species distribution in the local environment as well as their diverse abilities to persist in the human respiratory tract.

Yu Pang ${ }^{1,5}$, Yaoju Tan ${ }^{2,5}$, Jin Chen ${ }^{3,5}$, Yanming $\mathrm{Li}^{4,5}$, Huiwen Zheng ${ }^{1}$, Yuanyuan Song ${ }^{1}$ and Yanlin Zhao ${ }^{1}$

${ }^{1}$ National Center for Tuberculosis Control and Prevention, Chinese Center for Disease Control and Prevention, Beijing, China. ${ }^{2}$ Dept of Clinical Laboratory, Guangzhou Chest Hospital, Guangzhou, China. ${ }^{3}$ Dept of Clinical Laboratory, Shanghai Pulmonary Hospital, Shanghai, China. ${ }^{4}$ Dept of Respiratory and Critical Care Medicine, Beijing Hospital, Beijing, China. ${ }^{5}$ These authors contributed equally.

Correspondence: Y. Zhao, National Center for Tuberculosis Control and Prevention, Chinese Center for Disease Control and Prevention, No. 155 Changbai Road, Changping District, Beijing 1022065, China. E-mail: zhaoyanlin@chinatb.org

Received: July 162016 | Accepted after revision: Nov 232016

Support statement: This study was supported by the Ministry of Science and Technology of the People's Republic of China (National Key Project 2013ZX0003-003). Funding information for this article has been deposited with the Open Funder Registry.

Conflict of interest: None declared.

\section{References}

1 Hoefsloot W, van Ingen J, Andrejak C, et al. The geographic diversity of nontuberculous mycobacteria isolated from pulmonary samples: an NTM-NET collaborative study. Eur Respir J 2013; 42: 1604-1613.

2 van Ingen J. Diagnosis of nontuberculous mycobacterial infections. Semin Respir Crit Care Med 2013; 34: 103-109.

3 Marras TK, Chedore P, Ying AM, et al. Isolation prevalence of pulmonary non-tuberculous mycobacteria in Ontario, 1997-2003. Thorax 2007; 62: 661-666.

4 Wang L, Zhang H, Ruan Y, et al. Tuberculosis prevalence in China, 1990-2010; a longitudinal analysis of national survey data. Lancet 2014; 383: 2057-2064.

5 Zhang Z, Pang Y, Wang Y, et al. Differences in risk factors and drug susceptibility between Mycobacterium avium and Mycobacterium intracellulare lung diseases in China. Int J Antimicrob Agents 2015; 45: 491-495.

6 Wang $\mathrm{X}, \mathrm{Li} \mathrm{H}$, Jiang G, et al. Prevalence and drug resistance of nontuberculous mycobacteria, northern China, 2008-2011. Emerg Infect Dis 2014; 20: 1252-1253.

7 Shao Y, Chen C, Song H, et al. The epidemiology and geographic distribution of nontuberculous mycobacteria clinical isolates from sputum samples in the eastern region of China. PLoS Negl Trop Dis 2015; 9: e0003623.

$8 \mathrm{Wu} \mathrm{J}$, Zhang Y, Li J, et al. Increase in nontuberculous mycobacteria isolated in Shanghai, China: results from a population-based study. PLoS One 2014; 9: e109736.

9 Aksamit TR, Philley JV, Griffith DE. Nontuberculous mycobacterial (NTM) lung disease: the top ten essentials. Respir Med 2014; 108: 417-425.

10 Prevots DR, Marras TK. Epidemiology of human pulmonary infection with nontuberculous mycobacteria: a review. Clin Chest Med 2015; 36: 13-34.

11 Falkinham JO 3rd. Environmental sources of nontuberculous mycobacteria. Clin Chest Med 2015; 36: 35-41.

12 Falkinham JO 3rd. Surrounded by mycobacteria: nontuberculous mycobacteria in the human environment. J Appl Microbiol 2009; 107: 356-367.

13 van Ingen $\mathrm{J}$, Boeree $\mathrm{MJ}$, Dekhuijzen $\mathrm{PN}$, et al. Environmental sources of rapid growing nontuberculous mycobacteria causing disease in humans. Clin Microbiol Infect 2009; 15: 888-893.

14 van Ingen J, Totten SE, Heifets LB, et al. Drug susceptibility testing and pharmacokinetics question current treatment regimens in Mycobacterium simiae complex disease. Int J Antimicrob Agents 2012; 39: 173-176. 